\title{
Sterile neutrinos influence on oscillation characteristics of active neutrinos at short distances in the generalized model of neutrino mixing
}

\author{
V. V. Khruschov ${ }^{*, \dagger} \$$ and S. V. Fomichev*,‡, \\ ${ }^{*}$ National Research Centre Kurchatov Institute, \\ Academician Kurchatov Place 1, Moscow, 123182 Russia \\ ${ }^{\dagger}$ Center for Gravitation and Fundamental Metrology, \\ VNIIMS, Ozernaya Str. 46, Moscow, 119361 Russia \\ ${ }^{\ddagger}$ Moscow Institute of Physics and Technology (State University), \\ Institutskii Lane 9, Dolgoprudnyi, Moscow Region, 141700 Russia \\ \$khruschov_vv@nrcki.ru and \\ 『fomichev_sv@nrcki.ru
}

\begin{abstract}
A phenomenological model with active and sterile neutrinos is used for calculations of neutrino oscillation characteristics at the normal mass hierarchy of active neutrinos. Taking into account the contributions of sterile neutrinos, appearance and survival probabilities for active neutrinos are calculated. Modified graphical dependencies for the probability of appearance of electron neutrinos/antineutrinos in muon neutrino/antineutrino beams as a function of the ratio of the distance to the neutrino energy and other model parameters are obtained. It is shown that in the case of a certain type mixing between active and sterile neutrinos it is possible to clarify some features of the anomalies of neutrino data at short distances. A new parametrization for a particular type mixing matrix of active and sterile neutrinos that takes into account the additional sources of $\mathrm{CP}$ violation is used. The comparison with the existing experimental data is performed and, with using this knowledge, the estimates of some model parameters are found. The theoretical results obtained for mixing of active and sterile neutrinos can be applied for interpretation and prediction of results of ground-based experiments on search of sterile neutrinos as well as for the analysis of some astrophysical data.
\end{abstract}

PACS numbers: $12.10 . \mathrm{Kt}, 12.90 .+\mathrm{b}, 14.60 . \mathrm{Pq}, 14.60 . \mathrm{St}$

Keywords: Neutrino oscillations; Sterile neutrinos; CP-violation; Short-baseline neutrino anomalies 


\section{INTRODUCTION}

It is well known that oscillations of solar, atmospheric, reactor and accelerator active neutrinos (AN) can be attributed to mixing of three mass states of neutrinos. The mixing of these states 1 puts into operation with the Pontecorvo-Maki-Nakagawa-Sakata matrix $U_{\mathrm{PMNS}} \equiv U \equiv V P$, so that $\psi_{a}^{L}=\sum_{i} U_{a i} \psi_{i}^{L}$, where $\psi_{a, i}^{L}$ are left chiral fields with flavor $a$ or mass $m_{i}, a=\{e, \mu, \tau\}$ and $i=\{1,2,3\}$. For three active neutrinos, the matrix $V$ is expressed in the standard parametrization $\underline{2}$ via the mixing angles $\theta_{i j}$ and the CP-phase, namely, the phase $\delta \equiv \delta_{\mathrm{CP}}$ associated with $\mathrm{CP}$ violation in the lepton sector for Dirac or Majorana neutrinos, and $P=\operatorname{diag}\left\{1, e^{i \alpha}, e^{i \beta}\right\}$, where $\alpha \equiv \alpha_{\mathrm{CP}}$ and $\beta \equiv \beta_{\mathrm{CP}}$ are phases associated with $\mathrm{CP}$ violation only for Majorana neutrinos. In the atmospheric, solar, reactor and accelerator oscillation experiments it is impossible to measure $\alpha_{\mathrm{CP}}$ and $\beta_{\mathrm{CP}}$ and attribute neutrinos to Majorana or Dirac type of particles. Meanwhile, obtained experimental results made it possible to establish a breaking of conservation for lepton numbers $L_{e}, L_{\mu}$ and $L_{\tau}$.

With the help of high-precision experimental data, the values of the mixing angles and the differences of the neutrino masses in square $\Delta m_{21}^{2}$ and $\Delta m_{31}^{2}$ were found ${ }^{2,3}$ (where $\Delta m_{i j}^{2}=m_{i}^{2}-$ $m_{j}^{2}$ ). For these neutrino masses in square differences only absolute value of $\Delta m_{31}^{2}$ is known, therefore, the absolute values of the neutrino masses can be ordered by two ways, namely, as $m_{1}<m_{2}<m_{3}$ or $m_{3}<m_{1}<m_{2}$. These cases are called as normal hierarchy (NH) and as inverse hierarchy $(\mathrm{IH})$ of the neutrino mass spectrum, respectively. Including nonzero neutrino masses leads to the Minimally Extended Standard Model (MESM) instead of the Standard Model $(\mathrm{SM})$. Although the value of CP-phase $\delta_{\mathrm{CP}}$ is not yet definitively determined experimentally, in a number of papers its estimate was obtained (for example, see Refs. 3 - 6 ). For the NH-case of the mass spectrum of active neutrinos we have $\sin \delta_{\mathrm{CP}}<0$ and $\delta_{\mathrm{CP}} \approx-\pi / 2$. If we take into account the restrictions on the sum of the neutrino masses from cosmological observations $\frac{7}{\underline{ }}$ and the results of the $\mathrm{T} 2 \mathrm{~K}$ experiment $\underline{\underline{6}}$, then the $\mathrm{NH}$-case of the neutrino mass spectrum turns out to be preferable. So, in carrying out further numerical calculations we restrict ourselves to the NH-case only, assuming $\delta_{\mathrm{CP}} \equiv \delta_{1}=-\pi / 2$.

At the same time, there are indications to anomalies of neutrino fluxes for some processes that can not be explained with using oscillation parameters only for three active neutrinos. These anomalies include LSND (or accelerator) anomaly $\underline{\underline{8}} \underline{\underline{11}}$, reactor $\underline{\underline{12}}-\underline{17}$ and gallium (calibration) $)^{18} \underline{\underline{20}}$ anomalies. The anomalies manifest themselves at short distances (more precisely, at distances $L$ such that the numerical value of the parameter $\Delta m^{2} L / E$, where $E$ is the neutrino energy, is of the order of unity). In the LSND anomaly, an excess of the electron antineutrinos in beams of muon 
antineutrinos in comparison with the expected value according to the MESM is observed. Similar results were observed in the MiniBooNE experiments for electron neutrinos and antineutrinos $\frac{10}{11}$. Deficit of reactor electron antineutrinos at short distances is called as reactor anomaly, while the deficit of electron neutrinos from a radioactive source occurred at calibration of detectors for the SAGE and GALLEX experiments is commonly called as gallium anomaly. In other words, data on anomalies refer to both the appearance of electron neutrinos or antineutrinos in beams of muon neutrinos or antineutrinos, respectively, and to the disappearance of electron neutrinos or antineutrinos. These three types of the shot-baseline (SBL) neutrino anomalies, for which there are indications at present, may be explained by the existence of one or two new neutrinos that do not interact directly with the gauge bosons of the SM, that is sterile neutrinos (SN). The characteristic mass scale of sterile neutrino used for explanation of the SBL anomalies is about $1 \mathrm{eV}$.

Today, in addition to SN, other new neutral particles are present in many models that are beyond the framework of SM such as supersymmetric models, grand unification theories, different phenomenological models, etc. The using of some new particles is associated with necessity to explain a number of phenomena in cosmology, astrophysics and particle physics that are difficult to explain in terms of SM particles only. As an example we point to neutral fermions with large masses (heavy neutrinos (HN)) and dark matter particles (DMP), of which, perhaps, dark matter (DM) consists, at that SN and HN can be components of DMP. Almost all characteristics of DMP remain unknown now 21,22 . DM is not of the baryon nature and, in its turn, probably consists of cold dark matter (CDM), warm dark matter (WDM) and hot dark matter (HDM). Models with a multicomponent dark matter are often used to describe various structures with different scales in the Universe. More details about properties of DMP, including SN and HN, can be found in numerous papers (see, for example, Refs. 22 26).

The mass values of SN, HN and DMP belong to a wide range from $10^{-6} \mathrm{eV}$ to $10^{16} \mathrm{GeV}^{27} \underline{29}$. Their interactions with MESM particles can be realized by means of new scalar, pseudoscalar or vector bosons, in some cases new vector bosons can be mixed with the photon and/or the $Z$-boson. The latest data obtained on studying the Cosmic Microwave Background with the help of a number of cosmological models lead to a restriction on the number of new relativistic particles that were in the thermodynamic equilibrium in the era of plasma recombination in the early Universe $\underline{30}-\underline{32}$. So, it is convenient to associate a mass scale between light and heavy neutrinos with the values of temperature, which are typical for the recombination epoch and belong to the eV-range. The maximum value of this temperature is $R_{\infty} h c \approx 13.6 \mathrm{eV}$ ( $R_{\infty}$ is the Rydberg constant). That is, neutrinos with masses smaller than or equal to $13.6 \mathrm{eV}$ may be called as light neutrinos (LN), while 
neutrinos with masses large than $13.6 \mathrm{eV}$ may be called as heavy neutrinos (HN). Then the LN will include well-known active neutrinos $\nu_{e}, \nu_{\mu}$ and $\nu_{\tau}$, and also perhaps new light SN. If one takes into account the possibility of realization of non-standard models, where sterile LN did not thermalize in the plasma of the early Universe, then LN effect in cosmological data should be suppressed (see, for example, Refs. 33, 34). HN with masses less than half of the mass of the $Z$-boson are SN, while more heavy neutrinos, in principle, can interact directly with $Z$-boson, as, for example, a hypothetical heavy neutrino from the fourth generation or WIMPs.

At present, intensive searches are carried out for light SNs with masses of the order of $1 \mathrm{eV}$, which are proposed for explanation of the SBL anomalies. It is expected that in the coming several years it will be possible to confirm or deny the existence of such anomalies and light SN (see, for example, Refs. 23,28,35 38). Besides, HN with masses from several keV to several $\mathrm{TeV}$ are often used to explain some astrophysical data $22,39,40$. As the existence of SN and HN goes beyond MESM, there have been proposed phenomenological models for prediction of their characteristics, as well as effects due to them (see, for example, Refs. 4, 24, 41 44). For instance, in Ref. 45 the effect of increasing of sterile neutrinos yield in a high-density medium when the ratio of the number of neutrons to the number of protons approaches to two was considered in detail. This effect can have impact on the characteristics of fluxes of active neutrinos in supernovae $\frac{46,47}{}$. Phenomenological models with additional SN and HN are usually denoted as $(3+N)$ models, or, in detail, as $(k+3+n+m)$ models, where $k$ is the number of new neutrinos with masses less than masses of active neutrinos, and $n$ and $m$ are the numbers of new neutrinos with masses higher and considerably higher, respectively, than masses of the active neutrinos $1,23,43,44,48-51$.

In this paper, we present a phenomenological $(3+3)$ model ${ }^{52}$ with three active neutrinos and three SN and consider the effects of SN, which appear in oscillation characteristics of active neutrinos with energies of the order of $\mathrm{MeV}$ or dozens of $\mathrm{MeV}$ at small distances (of the order of several meters or tens of meters, corresponding to the SBL experiments). In Section $\amalg$, the main concepts of our $(3+3)$ model (to be exact, the $(3+1+2)$ model) based on the results obtained earlier $\underline{\underline{4}} \underline{\underline{4}}$ are given in detail, while in Section III the results of detailed calculations of the oscillation characteristics of active neutrinos at small distances with account of effects of SN are presented. Calculations were carried out with the use of new parametrization for the generalized mixing matrix of LN and $\mathrm{HN}$ at selected test values of the model parameters. By introducing additional CP-phases in the framework of our model, one can explain asymmetry of the yield of electron neutrinos and antineutrinos in beams of muon neutrinos and antineutrinos, respectively. The comparison is made with the simplest $(3+1)$ model, where in the main approximation the asymmetry is absent. The results 
of the performed SBL experiments are taken into account and on this basis tentative estimates of some model parameters are made. In the final Section IV it is noted that the obtained results can help to explain the available experimental data on the LSND and MiniBooNE anomaly and reactor and gallium anomalies, as well as to interpret both expected data of SBL experiments on the search of sterile neutrinos and some astrophysical data.

\section{BASIC CONCEPTS OF THE PHENOMENOLOGICAL $(3+1+2)$ MODEL OF LIGHT AND HEAVY NEUTRINOS}

The $(3+N)$ or $(3+m+n)$ phenomenological neutrino models can be used to describe the SBL anomalies, as well as some astrophysical data, where $N=m+n$ is the number of additional neutrinos, which, in principle, can be arbitrary (see, for example, Refs. 1, 23, $, 51,52$ ). It is desirable that the number of new neutrinos would be minimal, so the most common are the $(3+1)$ and $(3+2)$ models. However, if we apply the principle of extended symmetry of weak interactions, then, for example, for the left-right symmetry it is necessary in this case to consider $(3+3)$ models $\underline{4} \underline{42}-\underline{44}$. So, below we consider a $(3+1+2)$ model that can be used to describe effects of light and heavy $\mathrm{SN}$. This model includes three active neutrinos $\nu_{a}(a=e, \mu, \tau)$ and three new neutrinos: a sterile neutrino $\nu_{s}$, a hidden neutrino $\nu_{h}$ and a dark neutrino $\nu_{d}$. We use a characteristic order of mass values of $\nu_{s}, \nu_{h}$ and $\nu_{d}$ as well as a new parametrization of the mixing matrix, so our model can be considered as a generalization of the $(3+3)$ model, which was studied in Refs. $4,43,44$.

In order to take into account the contributions of light and heavy SN to the oscillation characteristics of active neutrinos, it will be considered the $6 \times 6$ mixing matrix, which can be called as the generalized mixing matrix $U_{\text {mix }}$, or the generalized Pontecorvo-Maki-Nakagawa-Sakata matrix $U_{\text {GPMNS }} \equiv U_{\text {mix }}{ }_{4}, 44$. This matrix can be represented as the matrix product $V P$, where $P$ is a diagonal matrix with Majorana CP-phases $\phi_{i}, i=1, \ldots, 5$, namely, $P=\operatorname{diag}\left\{1, e^{i \phi_{1}}, \ldots, e^{i \phi_{5}}\right\}$. Below we will consider only the particular type of matrix $V$. Keeping continuity of the notations, we will denote fifteen Dirac CP-phases as $\delta_{i}$ and $\kappa_{j}$, and twenty one mixing angles as $\theta_{i}$ and $\eta_{j}$, where $\delta_{1} \equiv \delta_{\mathrm{CP}}, \theta_{1} \equiv \theta_{12}, \theta_{2} \equiv \theta_{23}$ and $\theta_{3} \equiv \theta_{13}$.

For the compactness of the formulas, we introduce the symbols $h_{s}$ and $h_{i^{\prime}}$ for generalized left flavor fields and generalized left mass fields, respectively. As $s$ we will use a set of indices that allocate $\nu_{s}, \nu_{h}$ and $\nu_{d}$ fields among $h_{s}$, and as $i^{\prime}$ we will use a set of indices 4,5 and 6 . The common 
$6 \times 6$ mixing matrix $U_{\text {mix }}$ can then be expressed through $3 \times 3$ matrices $R, T, V$ and $W$ as follows

$$
\left(\begin{array}{c}
\nu_{a} \\
h_{s}
\end{array}\right)=U_{\text {mix }}\left(\begin{array}{c}
\nu_{i} \\
h_{i^{\prime}}
\end{array}\right) \equiv\left(\begin{array}{cc}
R & T \\
V & W
\end{array}\right)\left(\begin{array}{c}
\nu_{i} \\
h_{i^{\prime}}
\end{array}\right) .
$$

We represent the matrix $R$ in the form of $R=U_{\mathrm{PMNS}}+\Delta U_{\mathrm{PMNS}}$, where the matrix $\Delta U_{\mathrm{PMNS}}$, as well as the matrix $T$ in equation (1) should be small as compared with the matrix $U_{\text {PMNS }}$. For the convenience of quantitative estimates of arising corrections to mixing between active neutrinos due to $\mathrm{SN}$, we will put $\Delta U_{\mathrm{PMNS}}=-\epsilon U_{\mathrm{PMNS}}$, where $\epsilon$ is a small value, which can be presented as $\epsilon=1-\varkappa$. Then the matrix $R$ will be represented as $R=\varkappa U_{\text {PMNS }}$, that is, it will be proportional to the known unitary $3 \times 3$ mixing matrix of active neutrinos $\left(U_{\mathrm{PMNS}} U_{\mathrm{PMNS}}^{+}=I\right)$. Then we will use the notation $U_{\mathrm{PMNS}} \equiv U$.

Thus, when choosing the appropriate normalization, the active neutrinos mix, as it should be in the MESM, according to Pontecorvo-Maki-Nakagawa-Sakata matrix. Bearing in mind that, in accordance with data available due to astrophysical and laboratory measurements, the mixing between active and new neutrinos is small, we choose the matrix $T$ as $T=\sqrt{1-\varkappa^{2}} a$, where $a$ is an arbitrary unitary $3 \times 3$ matrix, that is, $a a^{+}=I$. The matrix $U_{\text {mix }}$ can now be written in the form of

$$
U_{\text {mix }}=\left(\begin{array}{cc}
R & T \\
V & W
\end{array}\right) \equiv\left(\begin{array}{cc}
\varkappa U & \sqrt{1-\varkappa^{2}} a \\
\sqrt{1-\varkappa^{2}} b U & \varkappa c
\end{array}\right),
$$

where $b$ is also an arbitrary unitary $3 \times 3$ matrix $\left(b b^{+}=I\right)$, and $c=-b a$. With these conditions, the matrix $U_{\text {mix }}$ will be unitary $\left(U_{\text {mix }} U_{\text {mix }}^{+}=I\right)$. For the matrix $U_{\text {mix }}$ we will consider, taking into account additional physical reasons, only some particular cases, but not the most common form. In particular, we will use the following matrices $a$ and $b$ :

$$
a=\left(\begin{array}{rcr}
\cos \eta_{2} & \sin \eta_{2} & 0 \\
-\sin \eta_{2} & \cos \eta_{2} & 0 \\
0 & 0 & e^{-i \kappa_{2}}
\end{array}\right), \quad b=-\left(\begin{array}{rcr}
\cos \eta_{1} & \sin \eta_{1} & 0 \\
-\sin \eta_{1} & \cos \eta_{1} & 0 \\
0 & 0 & e^{-i \kappa_{1}}
\end{array}\right)
$$

where $\kappa_{1}$ and $\kappa_{2}$ are mixing phases for active and sterile neutrinos, whereas $\eta_{1}$ and $\eta_{2}$ are their mixing angles. The matrix $a$ in the form of equation (3) was proposed in Ref. 4 . In order to make our calculations more specific, we will use the following test values for new mixing parameters:

$$
\kappa_{1}=\kappa_{2}=-\pi / 2, \quad \eta_{1}=5^{\circ}, \quad \eta_{2}= \pm 30^{\circ},
$$

and assume that the small parameter $\epsilon$ satisfies at least the condition $\epsilon \lesssim 0.03$. 
Note that the mixing matrix in the form of equation (2) is more general in comparison with the mixing matrix $\widetilde{U}$ that was proposed and used in Ref. 4. Indeed, in that paper the $3 \times 3$ matrix $c$ was reduced to a diagonal matrix, in fact to a phase factor $e^{i \phi}$, and also there was no additional independent mixing angle $\eta_{1}$, since the matrix $b$ was chosen proportional to matrix $a^{+}$. In the version of neutrino mixing discussed here, that corresponds to equations (21) and (3), there are more possibilities to describe the various contributions of SN.

The neutrino masses will be given by a normally ordered set of values $\{m\}=\left\{m_{i}, m_{i^{\prime}}\right\}$. For active neutrinos we will use the neutrino mass estimations, which were proposed in Refs. 4, 43, 47 for $\mathrm{NH}$-case (in units of eV) and which do not contradict to the known experimental data:

$$
m_{1} \approx 0.0016, \quad m_{2} \approx 0.0088, \quad m_{3} \approx 0.0497
$$

The values of the mixing angles $\theta_{i j}$ of active neutrinos that determine the Pontecorvo-MakiNakagawa-Sakata mixing matrix will be taken from relations $\sin ^{2} \theta_{12} \approx 0.297, \sin ^{2} \theta_{23} \approx 0.425$ and $\sin ^{2} \theta_{13} \approx 0.0215$, which are obtained from the processing of experimental data and given in Ref. 2 .

In what follows we consider two variants of the $(3+1+2)$ model, which differ by the sterile neutrino mass $m_{4}$. The values of this mass are selected on the basis of experimental results of MiniBooNE, NEOS, DANSS and Neutrino-4 experiments 11 15-17 , which point to the values of 0.2 , 1.14, 1.18 and $2.65 \mathrm{eV}$, respectively, as at the best fit for sterile neutrino mass. Taking into account the considerable dispersion of these results, we choose two $m_{4}$ values, 0.55 and $1.1 \mathrm{eV}$, as possible test values in the framework of our model.

On the other hand, if to select, together with the mass value of the light neutrino $m_{4} \sim 1 \mathrm{eV}$, the mass value $m_{6}$ associated with particle $\nu_{d}$ as about $10 \mathrm{keV}$, it becomes possible to explain the appearance of anomalies at short distances in neutrino data 50 , as well as the registration of the line $3.55 \mathrm{keV}$ in the gamma spectra of some astrophysical sources $\underline{53}-\underline{55}$. Note that sterile neutrinos with masses of the order of $1 \mathrm{keV}$ are also used for interpretation of some astrophysical data, so we choose the mass value $m_{5}$ associated with particles $\nu_{h}$ as about $1 \mathrm{keV}$. Thus, let us to consider the mass option, which can be designated as "Light Mass Option" (LMO), in two variants:

$$
\begin{gathered}
\{m\}_{\mathrm{LMO} 1}=\left\{1.1,1.5 \times 10^{3}, 7.5 \times 10^{3}\right\} . \\
\{m\}_{\mathrm{LMO} 2}=\left\{0.55,1.5 \times 10^{3}, 7.5 \times 10^{3}\right\} .
\end{gathered}
$$

The probability amplitudes for propagation of neutrino flavors can be found by solution of well-known equations (see, for example, Ref. 4,, 57 ). Moreover, with the help of these equations, 
analytical expressions for transition probabilities between different flavors in neutrino/antineutrino beams in vacuum as a function of the distance from the neutrino source can be obtained $\stackrel{51}{ }$, which are also used in the current paper in calculations to control the results obtained by numerical solution of the equations.

For three active neutrinos, almost always ultrarelativistic, these equations have the form

$$
i \partial_{r}\left(\begin{array}{c}
a_{e} \\
a_{\mu} \\
a_{\tau}
\end{array}\right)=H\left(\begin{array}{c}
a_{e} \\
a_{\mu} \\
a_{\tau}
\end{array}\right),
$$

where the matrix $H$ is expressed using the matrix $U_{\mathrm{PMNS}} \equiv U$ in the form of

$$
H=\frac{U}{2 E}\left(\begin{array}{ccc}
m_{1}^{2}-m_{0}^{2} & 0 & 0 \\
0 & m_{2}^{2}-m_{0}^{2} & 0 \\
0 & 0 & m_{3}^{2}-m_{0}^{2}
\end{array}\right) U^{+} .
$$

Here $m_{0}$ is the smallest value among three neutrino masses $m_{1}, m_{2}$ and $m_{3}$, and $E$ is the neutrino energy. In what follows, as a basic case it will be used here the simplest conventional approach for neutrino oscillations that is based on the plane-wave neutrino states. The more consistent approach with wave packets of the neutrino states (see, e.g., Ref. 58), which takes into account some coherence limitations, can also be considered in further elsewhere.

In the plane-wave approximation, the neutrinos possess equal momentums that leads to the diagonal neutrino energy matrix $\Delta_{E}$ in the form of

$$
\Delta_{E}=\operatorname{diag}\left\{E_{1}-E_{0}, E_{2}-E_{0}, \ldots, E_{6}-E_{0}\right\},
$$

where $E_{i}=\sqrt{p^{2}+m_{i}^{2}}, m_{i}(i=1,2, \ldots 6)$ are the neutrino masses and $m_{0}$ is the smallest mass among $m_{i}$. The momentum $p$ can be related to the energy $E \approx p$ of ultrarelativistic active neutrinos. In the ultrarelativistic limit for all neutrinos, in place of the matrix $\Delta_{E}$ it is possible to use the matrix $\Delta_{m^{2}}$ of the differences of the squares of neutrino masses, which in the general case of $3+N$ flavors is defined as

$$
\Delta_{m^{2}}=\operatorname{diag}\left\{m_{1}^{2}-m_{0}^{2}, m_{2}^{2}-m_{0}^{2}, \ldots, m_{3+N}^{2}-m_{0}^{2}\right\}
$$

Then it is necessary to solve the following equations for neutrino propagation, similar to the equations (8) and (9) for active neutrinos:

$$
i \partial_{r}\left(\begin{array}{c}
a_{a} \\
a_{s}
\end{array}\right)=\frac{U_{\mathrm{mix}}}{2 E} \Delta_{m^{2}} U_{\mathrm{mix}}^{+}\left(\begin{array}{c}
a_{a} \\
a_{s}
\end{array}\right),
$$


where $U_{\text {mix }}$ is the unitary $6 \times 6$ neutrino mixing matrix given by equations (2)-(3) and $r=r_{a} \approx c t$ is the distance traveled by active neutrinos (it was assumed above that $c=1$ ). For antineutrinos, the equations have the form

$$
i \partial_{r}\left(\begin{array}{c}
a_{\bar{a}} \\
a_{\bar{s}}
\end{array}\right)=\frac{U_{\mathrm{mix}}^{*}}{2 E} \Delta_{m^{2}} U_{\mathrm{mix}}^{T}\left(\begin{array}{c}
a_{\bar{a}} \\
a_{\bar{s}}
\end{array}\right),
$$

where $*$ means complex conjugation. Solving these equations for certain values of the parameters, one can find the survival probabilities, and also probabilities of appearance and disappearance of neutrinos or antineutrinos of any flavor as functions of the neutrino/antineutrino energy and the distance from the source.

From equations (11)-(13), analytical expressions for the probabilities of transitions between different flavors of neutrinos/antineutrinos in vacuum as a function of the distance $L$ from the source can be obtained. If $\widetilde{U} \equiv U_{\text {mix }}$ is a generalized $6 \times 6$ mixing matrix in the form given by equation (2), and if we use the notation $\Delta_{k i} \equiv \Delta m_{i k}^{2} L /(4 E)$, then, following by Ref. 51, it is possible to calculate the transition probabilities from $\nu_{\alpha}$ to $\nu_{\alpha^{\prime}}$ or from $\bar{\nu}_{\alpha}$ to $\bar{\nu}_{\alpha^{\prime}}$ by the formula

$$
\begin{aligned}
P\left(\nu_{\alpha}\left(\bar{\nu}_{\alpha}\right) \rightarrow \nu_{\alpha^{\prime}}\left(\bar{\nu}_{\alpha^{\prime}}\right)\right)=\delta_{\alpha^{\prime} \alpha} & -4 \sum_{i>k} \operatorname{Re}\left(\widetilde{U}_{\alpha^{\prime} i} \widetilde{U}_{\alpha i}^{*} \widetilde{U}_{\alpha^{\prime} k}^{*} \widetilde{U}_{\alpha k}\right) \sin ^{2} \Delta_{k i} \\
& \pm 2 \sum_{i>k} \operatorname{Im}\left(\widetilde{U}_{\alpha^{\prime} i} \widetilde{U}_{\alpha i}^{*} \widetilde{U}_{\alpha^{\prime} k}^{*} \widetilde{U}_{\alpha k}\right) \sin 2 \Delta_{k i},
\end{aligned}
$$

where the upper sign $(+)$ corresponds to neutrino transitions $\nu_{\alpha} \rightarrow \nu_{\alpha^{\prime}}$, while the lower sign (-) corresponds to antineutrino transitions $\bar{\nu}_{\alpha} \rightarrow \bar{\nu}_{\alpha^{\prime}}$. Note, that the flavor indices $\alpha$ and $\alpha^{\prime}$ (also as summation indices $i$ and $k$ over massive states) apply to all neutrinos, that is to active, sterile and heavy neutrinos. Moreover, as follows from equation (14), the relation $P\left(\nu_{\alpha} \rightarrow \nu_{\alpha}\right) \equiv P\left(\bar{\nu}_{\alpha} \rightarrow \bar{\nu}_{\alpha}\right)$ is fulfilled exactly as a consequence of the CPT-invariance $\underline{\underline{ }}$.

To check the accuracy of the numerical results obtained on the basis of equations (12) and (13) taking into account the subtle effects of the possible existence of $\mathrm{SN}$ and $\mathrm{HN}$, calculations were also performed with the help of precise analytical expressions (14). Moreover, the probabilities of the processes of interest to us, namely, the processes of appearance of electron neutrinos/antineutrinos in a beam of muon neutrino/antineutrino depend only on the first two rows of the matrix $U_{\text {mix }}$ and can be explicitly written (see the Appendix). From expressions (A1)-(A8) it is clearly seen that some parameters of the model that were introduced before for the sake of generality, namely, the mixing phases $\kappa_{1}$ and $\kappa_{2}$, the mixing angle $\eta_{1}$ and the heavy neutrino mass $m_{6}$ are not involved in the processes of appearance of electron neutrinos/antineutrinos in a beam of muon neutrinos/antineutrinos. Only parameter $\epsilon$, masses $m_{4}$ and $m_{5}$ of two sterile/heavy neutrinos and the 
mixing angle $\eta_{2}$, along with the mixing parameters of three active neutrinos are responsible for these processes. This is also true for processes of survival of electron neutrinos/antineutrinos that are responsible for the gallium and reactor anomalies.

\section{NUMERICAL RESULTS FOR OSCILLATION CHARACTERISTICS OF ACTIVE NEUTRINOS WITH THE ACCOUNT OF THEIR MIXING WITH SN}

\section{A. Computation of oscillation characteristics at the test values of model parameters}

In this paper, we mainly focus on the possibility of describing, in the framework of the considered model, the anomalies found in the data of LSND and MiniBooNE experiments on oscillations of accelerator muon neutrinos and antineutrinos, which were subsequently tested and will still be tested in accelerator experiments $11,59,60$. It refers to data on the disappearance of muon neutrinos and antineutrinos and the appearance of electron neutrinos and antineutrinos in the processes $\nu_{\mu} \rightarrow \nu_{e}$ and $\bar{\nu}_{\mu} \rightarrow \bar{\nu}_{e}$. The ratio of the distance $L$ traveled by the neutrino before detection to the neutrino energy $E$ is typically a few meters per one $\mathrm{MeV}$. Attempts of the simultaneous description of all the data in these processes leads to difficulties. In particular, the problem associated with different values of the excess of the output of $\nu_{e}$ and $\bar{\nu}_{e}$ in the MiniBooNE experiment may be resolved under the condition of $\mathrm{CP}$ violation $\underline{\underline{61}} \underline{\underline{63}}$.

Note that the reactor and gallium anomalies manifesting themselves in neutrino data as the disappearance of electron neutrinos and antineutrinos can be described in the framework of the model considered in this paper with the new parametrization of the mixing matrix by selecting the value of the parameter $\epsilon$. To describe these anomalies, it is sufficient to choose the appropriate value less than unity and corresponding to the experimental data for the parameter $\varkappa=1-\epsilon$, which is present in our parametrization of the mixing matrix (see equation (2)) that naturally leads to the deficit of electron neutrinos and antineutrinos. The status of the reactor anomaly with allowance for the recently discovered excess of the number of antineutrinos in comparison with the model calculations in the $5 \mathrm{MeV}$ range and confirmation of the possible existence of a light SN with a mass of about $1 \mathrm{eV}$ see, for example, in Refs. 6466.

In the framework of our model the probabilities of the appearance of electron neutrinos and antineutrinos in accelerator beams of muon neutrinos and antineutrinos as a function of the ratio of the distance $L$ to the neutrino energy $E$ are shown in Figs. 1 2, respectively, for the mixing matrix in the form considered in this paper (equations (2)-(3)) and for the two mass options, namely, LMO1 (equation (6) ) and LMO2 (equation (17)). 

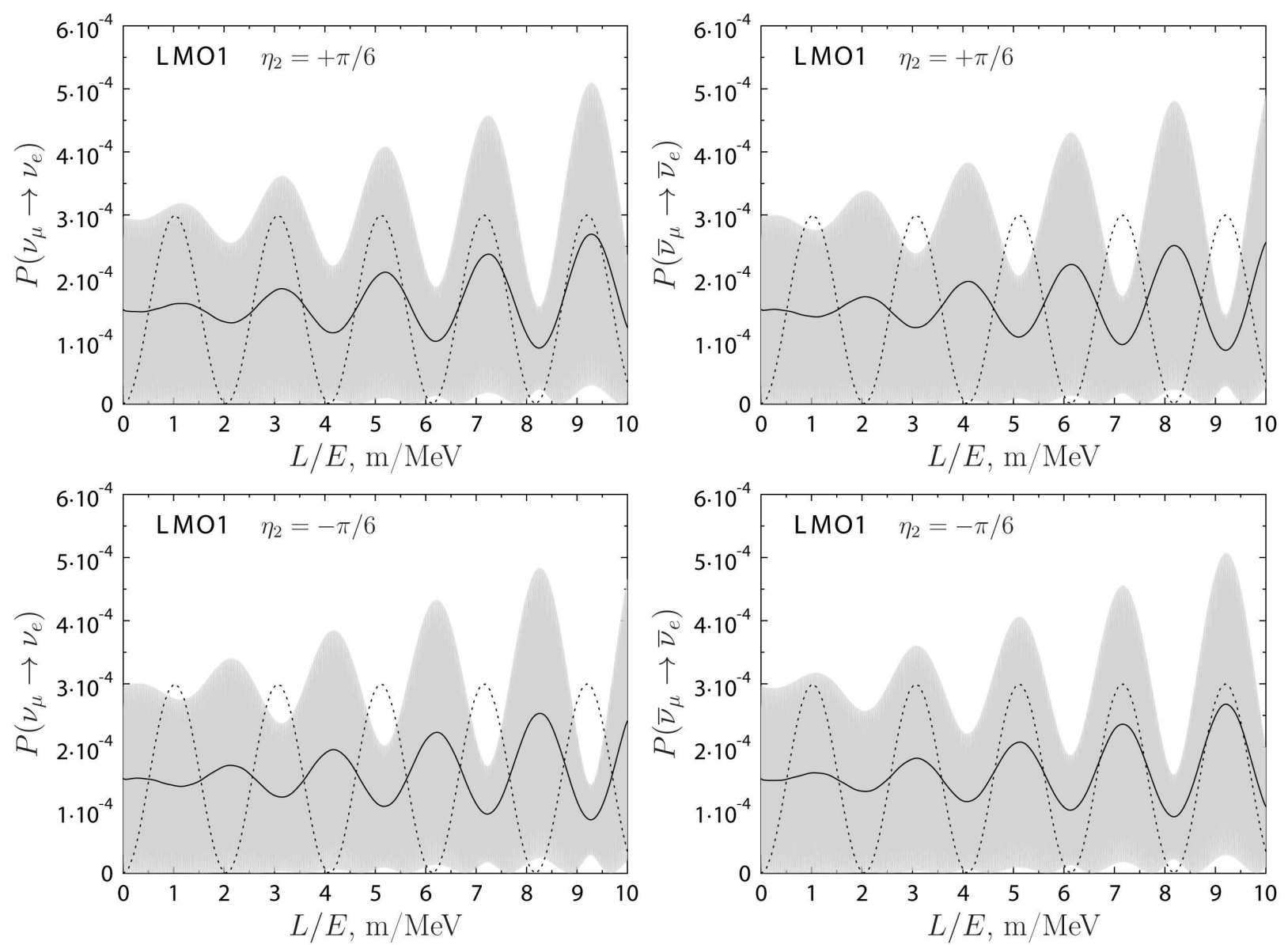

FIG. 1: The probability of appearance of electron neutrinos (left panels) and antineutrinos (right panels) versus the ratio of the distance $L$ from the source to the neutrino energy $E$ in the beams of muon neutrinos and antineutrinos, respectively. The value $\epsilon=0.01$ of the coupling constant of active and sterile neutrinos is taken for the case of the mixing matrix considered in this paper (equations (2)-(3)), for $\eta_{2}= \pm \pi / 6$, and for the LMO1 version (equation (6) ) of the mass values of sterile neutrinos. The gray region arises as a result of exact calculations due to fast oscillations caused by the presence in the model of fifth sterile neutrino with mass of the order of $1 \mathrm{keV}$, while the solid curves show probability values averaged over small-scale spatial oscillations. The dotted curves show probability values calculated in the simplest approximation of the $(3+1)$ model and the two-neutrino mixing with $\sin ^{2}(2 \theta)=0.0003$ and $\Delta m_{41}^{2}=1.21 \mathrm{eV}^{2}$.

Figure 1 shows the appearance probabilities of $\nu_{e}$ (left panels) and $\bar{\nu}_{e}$ (right panels) in the beams of $\nu_{\mu}$ and $\bar{\nu}_{\mu}$, respectively, as a function of the ratio of the distance $L$ to the neutrino energy $E$ at the value of the parameter $\epsilon=0.01$, for the parameter $\eta_{2}=+\pi / 6$ (upper panels) and $\eta_{2}=-\pi / 6$ (lower panels), and for the LMO1 case (equation 6) of neutrino mass distribution. Due to presence in the model of fifth neutrino with mass of the order of $1 \mathrm{keV}$, the exactly calculated curves are fast-oscillating functions of $L / E$ parameter with a smoothly oscillating envelopes that results to 

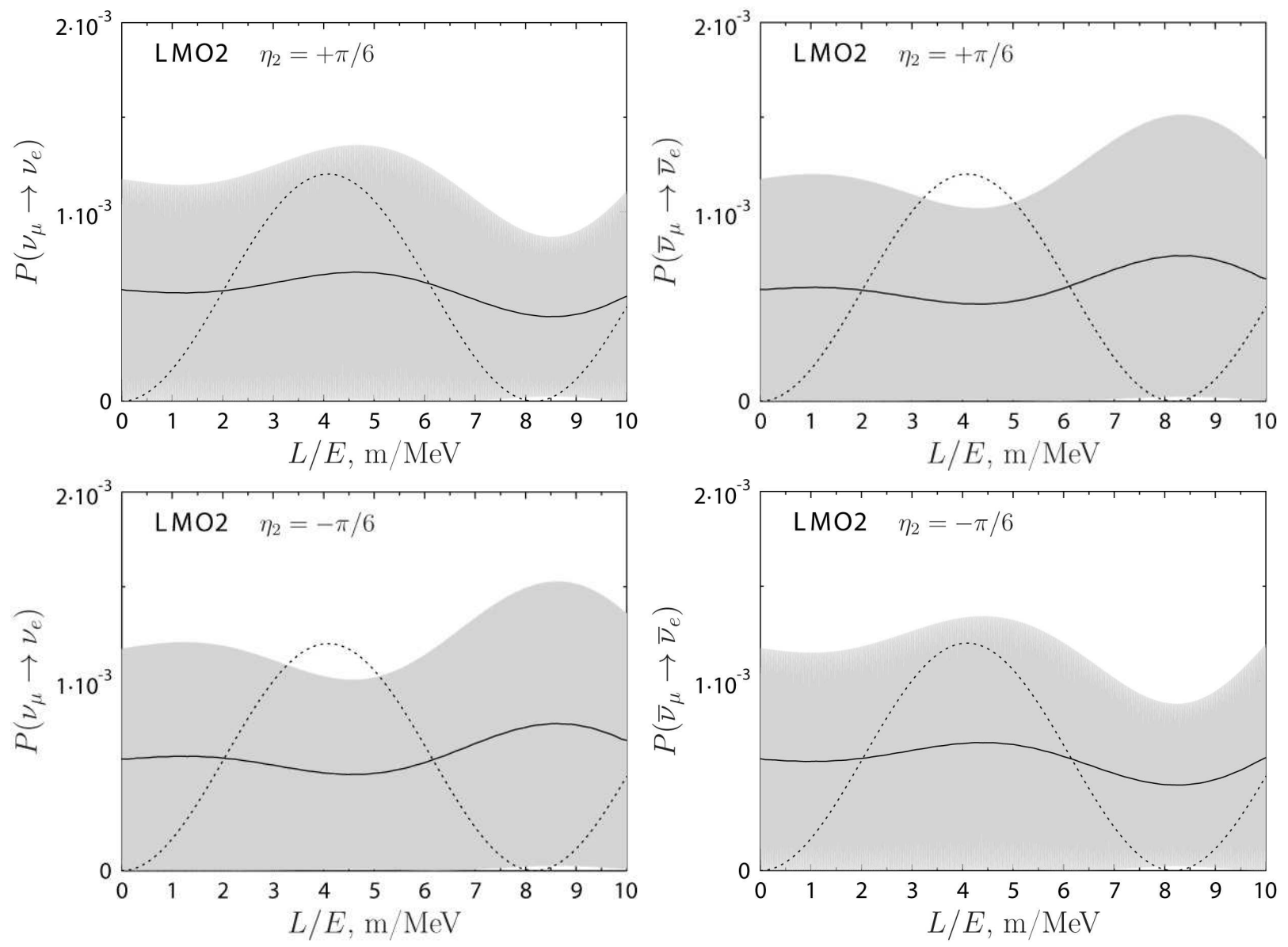

FIG. 2: The probability of appearance of electron neutrinos (left panels) and antineutrinos (right panels) versus the ratio of the distance $L$ from the source to the neutrino energy $E$ in the beams of muon neutrinos and antineutrinos, respectively. The value $\epsilon=0.02$ of the coupling constant of active and sterile neutrinos is taken for the case of the mixing matrix considered in this paper (equations (2)-(3)), for $\eta_{2}= \pm \pi / 6$, and for the LMO2 version (equation (7)) of the mass values of sterile neutrinos. The gray region arises as a result of exact calculations due to fast oscillations caused by the presence in the model of fifth sterile neutrino with mass of the order of $1 \mathrm{keV}$, while the solid curves show probability values averaged over small-scale spatial oscillations. The dotted curves show probability values calculated in the simplest approximation of the $(3+1)$ model and the two-neutrino mixing with $\sin ^{2}(2 \theta)=0.0012$ and $\Delta m_{41}^{2}=0.3 \mathrm{eV}^{2}$.

a grey region in Fig. 1. After averaging over these fast oscillations (solid curves in Fig. 1) that is quite reasonable from point of view of the experiment, the contribution of sterile neutrinos has the character of smooth oscillations. Furthermore, at $\eta_{2}=+\pi / 6$ (left upper panel) these oscillations for neutrinos are in phase with the oscillations, which are obtained with the help of the standard formula of the $(3+1)$ model for both the probability $P\left(\nu_{\mu} \rightarrow \nu_{e}\right)$ and $P\left(\bar{\nu}_{\mu} \rightarrow \bar{\nu}_{e}\right)$, that is by the formula $P=\sin ^{2}(2 \theta) \sin ^{2}\left(1.27 \Delta m_{41}^{2} L / E\right)$, where $L$ is the distance to the detector in $\mathrm{m}, E$ is the 

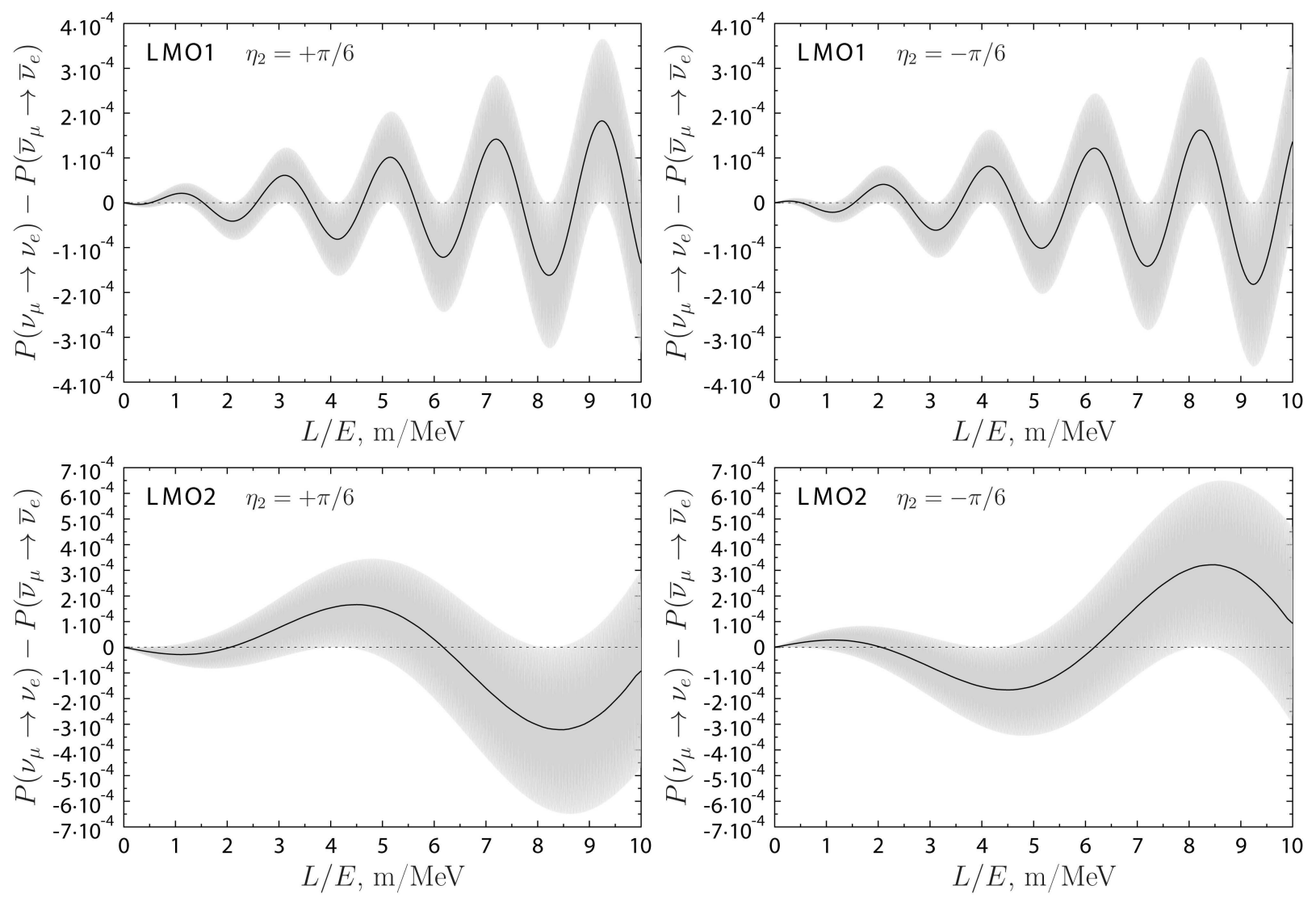

FIG. 3: The difference between the probabilities of the appearance of electron neutrinos and antineutrinos for cases of Fig. 1 (upper panels, LMO1) and Fig. 2 (lower panels, LMO2) versus the ratio of the distance $L$ from the source to the neutrino energy $E$ in the beams of muon neutrinos and antineutrinos, at $\eta_{2}=+\pi / 6$ (left panels) and $\eta_{2}=-\pi / 6$ (right panels). The gray region corresponds to exact calculations due to fast oscillations caused by the presence in the model of fifth sterile neutrino with mass of the order of $1 \mathrm{keV}$, while the solid curves show probability values averaged over small-scale spatial oscillations.

energy of neutrinos in $\mathrm{MeV}$, and $\Delta m_{41}^{2}$ is the neutrino mass-squared difference in $\mathrm{eV}^{2}$. On the other hand, for the same value of $\eta_{2}=+\pi / 6$ it is not the case for antineutrino oscillations (right upper panel), where the $(3+1)$ oscillations have a phase shift with respect to the $(3+1+2)$ oscillations. The situation is inverted at $\eta_{2}=-\pi / 6$ (lower panels). So we have the essential difference between oscillations in the frameworks of these models due to the additional source of $\mathrm{CP}$ violation in the $(3+1+2)$ model. This property of oscillations in the model with several neutrinos can be of important practical consequence while processing experimental data.

In Figure 2, the results for $P\left(\nu_{\mu} \rightarrow \nu_{e}\right)$ (right panels) and $P\left(\bar{\nu}_{\mu} \rightarrow \bar{\nu}_{e}\right)$ (left panels) are shown as a function of the ratio of the distance $L$ to the neutrino energy $E$ at the value of the parameter $\epsilon=0.02$, for the parameter $\eta_{2}=+\pi / 6$ (upper panels) and $\eta_{2}=-\pi / 6$ (lower panels), and for the 
LMO2 case (equation 7) of neutrino mass distribution. The correlation between the $(3+1)$ and $(3+1+2)$ oscillations is the same as in Figure1. There is the visual difference between the averaged probabilities (solid curves) of the appearance of neutrinos and antineutrinos. The scale of the effect for Fig. 1 and Fig. 2 differs several times in magnitude. Note that one light sterile neutrino with mass value of the order of $1 \mathrm{eV}$ is sufficient to explain the possible accelerator anomalies in neutrino data within the framework of the model considered in this paper.

Graphs of the difference of the averaged probabilities of appearance of electron neutrinos and antineutrinos (asymmetry) for the LMO1 and LMO2 cases are given in the upper and lower panels of Fig. 3. respectively, for two values of the parameter $\eta_{2}= \pm \pi / 6$ and for the parameter $\epsilon$ values that correspond to those in Figs. 1 and 2, An important result is an oscillating change of sign of this difference versus the $L / E$ value. Note that the sign of the asymmetry changes also when the sign of the parameter $\eta_{2}$ changes. When the neutrino energy increases (and $L / E$ decreases), detection of the asymmetry in the neutrino and antineutrino yields becomes much more difficult.

All these results are characteristic features of the considered version of $(3+1+2)$ model of active neutrinos with allowance made for SN contributions, and they can be used for interpreting the available experimental data and predicting the results of new experiments related to the problem of existence of sterile neutrinos.

\section{B. Estimates of some model parameters on the basis of currently available experimental} data

At present, experiments are carried out to make sure that SBL neutrino anomalies exist and can be explained due to the effects of one or a few SN64,67,68. The simple $(3+1)$ scheme, as a rule, is used for explanation of SBL anomalies, which includes three AN and one SN with a mass value of the order of $1 \mathrm{eV}$. In this case, the approximation with oscillations between only two neutrino mass states (the two-level approximation) is applied for calculation of probabilities of appearance and disappearance of AN with $\Delta m_{41}^{2}$ as a characteristic parameter. In the framework of this approximation, the probabilities of neutrino or antineutrino transitions are equal to each other. In our more complicated model the asymmetry between the probabilities of transitions of muon neutrinos to electron neutrinos and muon antineutrinos to electron antineutrinos take place (see Fig. 31). And so one of the main conclusions of the present paper is that the processing of experimental data for the neutrino transitions should be done separately from the antineutrino transitions. 


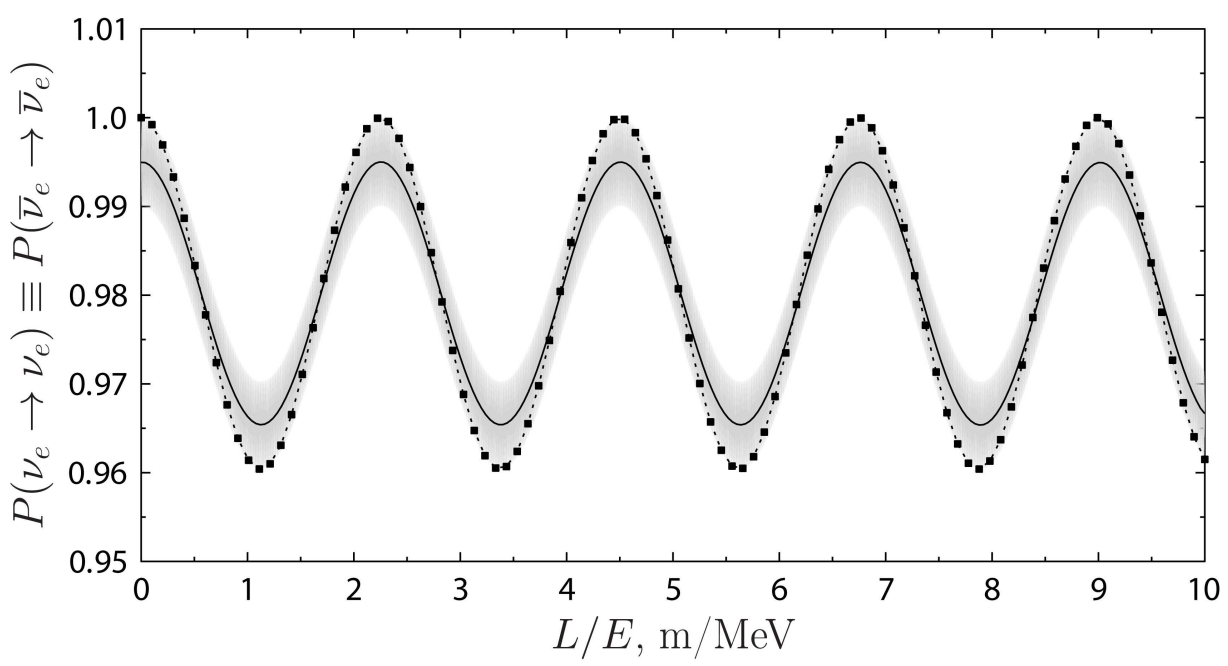

FIG. 4: The survival probability of electron neutrinos and antineutrinos versus the ratio of the distance $L$ from the source to the neutrino energy $E$ in the beams of electron neutrinos and antineutrinos. The value $\epsilon=0.005$ of the coupling constant of active and sterile neutrinos is taken for the case of the mixing matrix considered in this paper (equations (2)-(3)) with $\eta_{2}=\pi / 6$. The difference of masses squared with the light $\mathrm{SN}$ is equal to $\Delta m_{41}^{2}=1.1 \mathrm{eV}^{2}$. The gray region arises as a result of exact calculations due to fast oscillations caused by the presence in the model of fifth sterile neutrino with mass of the order of $1 \mathrm{keV}$, while the solid curve show probability values averaged over small-scale spatial oscillations. The dashed curve with black squares shows probability values calculated in the two-level approximation of the $(3+1)$ model and the two-neutrino mixing with $\sin ^{2}\left(2 \theta_{\mathrm{ee}}\right)=0.0396$ and $\Delta m_{41}^{2}=1.1 \mathrm{eV}^{2}$ from the global fit of the reactor antineutrino anomaly and gallium neutrino anomaly experimental data 64 .

There is considerable evidence for the SBL neutrino anomalies and their interpretation on the basic of the SN hypothesis (see, e.g. $\frac{67}{66}$ ). For a comparison with the available experimental data we prefer to use the updated global analysis of neutrino oscillations in the presence of eV-scale SN that has been done in Ref. 64. The results for $\Delta m_{41}^{2}, \sin ^{2}\left(2 \theta_{\mathrm{ee}}\right)$ and $\sin ^{2}\left(2 \theta_{\mu \mathrm{e}}\right)$ obtained in this global fit are used below for estimations of the light SN mass and the parameter $\epsilon$ by comparison with the results of numerical model calculations. Other model parameters will take test values as in the previous Section $\amalg$ IIA.

Figure 4 shows the survival probabilities of both $\nu_{e}$ and $\bar{\nu}_{e}$ in the beams of $\nu_{e}$ and $\bar{\nu}_{e}$, respectively, as a function of the ratio of the distance $L$ to the neutrino energy $E$ for the data obtained in the experiments for verification of the reactor and gallium anomalies. The interpolation of the experimental data with two-level approximation is given in the Fig. 4 by the standard (dashed) curve marked off by the black squares. The parameters $\Delta m_{41}^{2}$ and $\sin ^{2}\left(2 \theta_{\mathrm{ee}}\right)$ of this curve are picked out of "the domain with the star" on Figure 3 from Ref. 64. The other curves in Fig. 4 of 

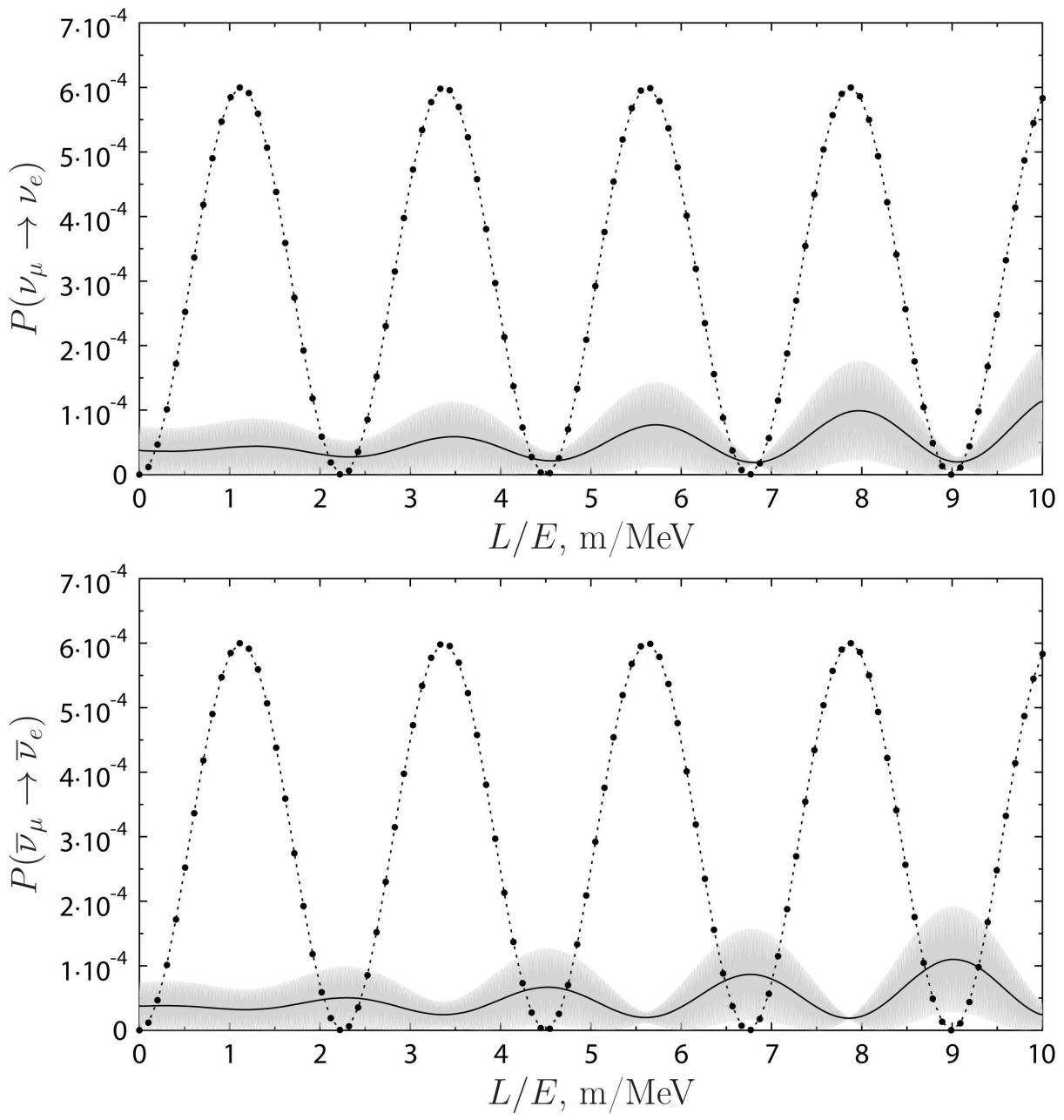

FIG. 5: The probability of appearance of electron neutrinos (upper panel) and antineutrinos (lower panel) versus the ratio of the distance $L$ from the source to the neutrino energy $E$ in the beams of muon neutrinos and antineutrinos, respectively. The value $\epsilon=0.005$ of the coupling constant of active and sterile neutrinos is taken for the case of the mixing matrix considered in this paper (equations (2)-(3) ) with $\eta_{2}=\pi / 6$. The difference of masses squared with the light $\mathrm{SN}$ is equal to $\Delta m_{41}^{2}=1.1 \mathrm{eV}^{2}$. The gray region arises as a result of exact calculations due to fast oscillations caused by the presence in the model of fifth sterile neutrino with mass of the order of $1 \mathrm{keV}$, while the solid curves show probability values averaged over small-scale spatial oscillations. The dashed curves with black circles show probability values calculated in the two-level approximation of the $(3+1)$ model and the two-neutrino mixing with $\sin ^{2}\left(2 \theta_{\mu \mathrm{e}}\right)=0.0006$ and $\Delta m_{41}^{2}=1.1 \mathrm{eV}^{2}$ from the global fit of the accelerator anomaly experimental data 64 .

the present paper are obtained by calculations in the framework of our model with $\Delta m_{41}^{2}=1.1 \mathrm{eV}^{2}$ and $\epsilon=0.005$.

Figure 5 shows the transition probabilities of $\nu_{\mu}$ in $\nu_{e}$ (upper panel) and $\bar{\nu}_{\mu}$ in $\bar{\nu}_{e}$ (lower panel) in the beams of $\nu_{\mu}$ and $\bar{\nu}_{\mu}$, respectively, for the data obtained in the experiments for verification of the 
accelerator anomaly. The interpolation of the experimental data with the two-level approximation is given in Fig. 5 with the standard (dashed) curve marked off by the black circles. The parameters $\Delta m_{41}^{2}$ and $\sin ^{2}\left(2 \theta_{\mu \mathrm{e}}\right)$ of this curve are picked out of the admissible region in the vicinity of "the combined domain" on Figure 4 (right panel) from Ref. 64 with the decay-at-rest and decay-at-flight data. The other curves in Fig. 5 of the present paper are obtained by our model calculations with the same values of parameters $\Delta m_{41}^{2}$ and $\epsilon$ as it was for the model curves on Fig. 4 ,

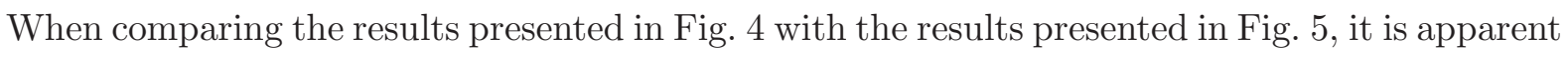
that the experimental data on the reactor and gallium SBL neutrino anomalies are represented well by the model calculations with the selected parameters while it is not the case for the accelerator SBL neutrino anomaly. In the latter case one can see the discrepancy between the experimental and the model calculated data. We interpret this point as the flaw in the data processing for the accelerator anomaly. This can be due in part to the joint processing of the neutrino and the antineutrino data. Besides, another problem is a significantly small value of the probability of transition $\nu_{\mu}\left(\bar{\nu}_{\mu}\right)$ to $\nu_{e}\left(\bar{\nu}_{e}\right)$, as is seen on the model curves in Fig. 5 .

\section{DISCUSSION AND CONCLUSIONS}

In this paper, we considered the phenomenological $(3+1+2)$ neutrino model with three active and three sterile neutrinos and examined the oscillation characteristics of active neutrinos in vacuum. The properties of these characteristics at the test values of the model parameters are numerically investigated. All calculations were performed for the case of a normal hierarchy of the mass spectrum of active neutrinos with allowance made for the possible violation of the CPinvariance in the lepton sector and for the value $-\pi / 2$ of the Dirac CP-phase in the $U_{\text {PMNS }}$ matrix. Graphical dependences of the probabilities of appearance of electron neutrinos and antineutrinos as a function of the ratio of the distance from the source of muon neutrinos and antineutrinos to the neutrino energy are given within two versions of the $(3+1+2)$ model, which differ in neutrino masses, namely, LMO1 and LMO2 (see Figs. 1 and 2). Besides, graphical representations of both survival and appearance probabilities of electron neutrinos and antineutrinos are given with the values of some model parameters obtained on the basis of comparison with the available experimental data (see Figs. 4 and 5 ).

The results obtained make it possible to interpret the experimental data on oscillations of neutrinos that admit the existence of the reactor and gallium anomalies (see Fig. 44) and to a lesser extent of the accelerator anomaly (see Fig. 5). But the great advantage of the considered model 
with regard to the accelerator anomaly is revelation of the asymmetry between the probabilities of the appearance of electron neutrinos and antineutrinos (see Fig. 3 and Fig. (5), which arises from the specific structure of the mixing matrix between AN and SN (see equations (10) and (2)). It is important that sufficiently large values of this asymmetry can be obtained with only one light SN within the LMO1 and LMO2 cases of the considered model. We note that the oscillatory character of the acceleration anomaly depends on the value of the lowest mass of SN. For example, if it is of the order of $1 \mathrm{eV}$, then there are oscillations at short distances. Results obtained for the probabilities of the appearance of electron neutrinos and antineutrinos (see Fig. (5) show the possible problems associated with observation of the LSND and MiniBooNE anomalies, in particular, the problem relevant to the $(\nu-\bar{\nu})$ asymmetry and the problem of a substantially small value of the transition probability of muon (anti)neutrinos in electron (anti)neutrinos. The selected value of the parameter $\epsilon$ can explain the reactor and gallium anomalies, as is seen in Fig. 4, but it is not the case for the acceleration anomaly (see Fig. 5). In a latter case, it would be a rather higher value of the parameter $\epsilon$. Notice that the gallium anomaly is free of the uncertainties of the neutrino energy spectrum.

In the near future, a number of ground-based experiments are planned, which are aimed at the search for sterile neutrinos $23,28,35,36$. Let us list some recent experimental results on the SN search. Two recent results with values of SN oscillation parameters, which both differ from results obtained in the other measurements and these results also differ between themselves. The NEUTRINO-4 result $\underline{38}$ is $\sin ^{2}\left(2 \theta_{e e}\right)=0.39, \Delta m_{41}^{2}=7.3 e V^{2}$. The MiniBooNE result 11 is $\sin ^{2}\left(2 \theta_{\mu e}\right)=0.84, \Delta m_{41}^{2}=0.039 \mathrm{eV}^{2}$. However the results of the DANSS and NEOS/Daya Bay experiments are independent from the theoretical Huber-Mueller flux calculation $\underline{12}, \underline{14}$ and from the $5 \mathrm{MeV}$ bump effect. The best-fit region of the combined analysis 66 of the combined fit of the NEOS/Daya Bay spectral ratio $\underline{\underline{15}}$ and the ratio of the spectra measured at 10.7 and 12.7 meters from the Kalinin reactor in the DANSS experiment $\underline{16}$ is $\left|U_{e 4}\right|^{2}=0.012 \pm 0.003, \Delta m_{41}^{2}=1.29 \pm 0.03 e V^{2}$. These values are close to the values of corresponding parameters of the $(3+1+2)$ model used in the present paper.

\section{Appendix A: Analytic expressions for $P\left(\nu_{\mu}\left(\bar{\nu}_{\mu}\right) \rightarrow \nu_{e}\left(\bar{\nu}_{e}\right)\right)$}

Here we give analytic expressions for the transition probabilities $\nu_{\mu}\left(\bar{\nu}_{\mu}\right) \rightarrow \nu_{e}\left(\bar{\nu}_{e}\right)$, which are obtained with using the formula (14). For convenience, the complete probability of each such transition is divided into the sum of partial contributions $P_{i k}$ corresponding to individual contributions 
with indexes $i>k$ to the sum of formula (14). One-type contributions are combined together in the expressions $P_{41+51}, P_{42+52}$ and $P_{43+53}$. In this way,

$$
P\left(\nu_{\mu}\left(\bar{\nu}_{\mu}\right) \rightarrow \nu_{e}\left(\bar{\nu}_{e}\right)\right)=P_{21}+P_{31}+P_{32}+P_{41+51}+P_{42+52}+P_{43+53}+P_{54},
$$

where

$$
\begin{aligned}
& P_{21}=(1-\epsilon)^{4} \sin \left(2 \theta_{12}\right) \cos ^{2} \theta_{13}\left\{\cos \left(2 \theta_{12}\right) \sin \theta_{13} \sin \left(2 \theta_{23}\right) \cos \delta_{C P}\right. \\
& \left.+\sin \left(2 \theta_{12}\right) \cos ^{2} \theta_{23}-\sin \left(2 \theta_{12}\right) \sin ^{2} \theta_{13} \sin ^{2} \theta_{23}\right\} \sin ^{2} \Delta_{12} \\
& \text { - } a(1-\epsilon)^{4} \sin \left(2 \theta_{12}\right) \sin \theta_{13} \cos ^{2} \theta_{13} \sin \theta_{23} \cos \theta_{23} \sin \delta_{C P} \sin \left(2 \Delta_{12}\right) \text {, } \\
& P_{31}=(1-\epsilon)^{4} \sin \left(2 \theta_{13}\right) \sin \theta_{23}\left\{\sin \left(2 \theta_{12}\right) \cos \theta_{13} \cos \theta_{23} \cos \delta_{C P}\right. \\
& \left.+\cos ^{2} \theta_{12} \sin \left(2 \theta_{13}\right) \sin \theta_{23}\right\} \sin ^{2} \Delta_{13} \\
& +a(1-\epsilon)^{4} \sin \left(2 \theta_{12}\right) \sin \theta_{13} \cos ^{2} \theta_{13} \sin \theta_{23} \cos \theta_{23} \sin \delta_{C P} \sin \left(2 \Delta_{13}\right) \text {, } \\
& P_{32}=-(1-\epsilon)^{4} \sin \left(2 \theta_{13}\right) \sin \theta_{23}\left\{\sin \left(2 \theta_{12}\right) \cos \theta_{13} \cos \theta_{23} \cos \delta_{C P}\right. \\
& \text { - } \left.\sin ^{2} \theta_{12} \sin \left(2 \theta_{13}\right) \sin \theta_{23}\right\} \sin ^{2} \Delta_{23} \\
& \text { - } a(1-\epsilon)^{4} \sin \left(2 \theta_{12}\right) \sin \theta_{13} \cos ^{2} \theta_{13} \sin \theta_{23} \cos \theta_{23} \sin \delta_{C P} \sin \left(2 \Delta_{23}\right) \text {, } \\
& P_{41+51}=-(1-\epsilon)^{2}\left(2 \epsilon-\epsilon^{2}\right) \sin \left(2 \eta_{2}\right)\left\{\cos ^{2} \theta_{12} \sin \left(2 \theta_{13}\right) \sin \theta_{23} \cos \delta_{C P}\right. \\
& \left.+\sin \left(2 \theta_{12}\right) \cos \theta_{13} \cos \theta_{23}\right\}\left(\sin ^{2} \Delta_{14}-\sin ^{2} \Delta_{15}\right) \\
& +a(1-\epsilon)^{2}\left(2 \epsilon-\epsilon^{2}\right) \sin \left(2 \eta_{2}\right) \cos ^{2} \theta_{12} \sin \theta_{13} \cos \theta_{13} \sin \theta_{23} \sin \delta_{C P} \\
& \times\left\{\sin \left(2 \Delta_{14}\right)-\sin \left(2 \Delta_{15}\right)\right\}, \\
& P_{42+52}=(1-\epsilon)^{2}\left(2 \epsilon-\epsilon^{2}\right) \sin \left(2 \eta_{2}\right)\left\{-\sin ^{2} \theta_{12} \sin \left(2 \theta_{13}\right) \sin \theta_{23} \cos \delta_{C P}\right. \\
& \left.+\sin \left(2 \theta_{12}\right) \cos \theta_{13} \cos \theta_{23}\right\}\left(\sin ^{2} \Delta_{24}-\sin ^{2} \Delta_{25}\right) \\
& +a(1-\epsilon)^{2}\left(2 \epsilon-\epsilon^{2}\right) \sin \left(2 \eta_{2}\right) \sin ^{2} \theta_{12} \sin \theta_{13} \cos \theta_{13} \sin \theta_{23} \sin \delta_{C P} \\
& \times\left\{\sin \left(2 \Delta_{24}\right)-\sin \left(2 \Delta_{25}\right)\right\}, \\
& P_{43+53}=(1-\epsilon)^{2}\left(2 \epsilon-\epsilon^{2}\right) \sin \left(2 \eta_{2}\right) \sin \left(2 \theta_{13}\right) \sin \theta_{23} \cos \delta_{C P}\left(\sin ^{2} \Delta_{34}-\sin ^{2} \Delta_{35}\right) \\
& \text { - } a(1-\epsilon)^{2}\left(2 \epsilon-\epsilon^{2}\right) \sin \left(2 \eta_{2}\right) \sin \theta_{13} \cos \theta_{13} \sin \theta_{23} \sin \delta_{C P} \\
& \times\left\{\sin \left(2 \Delta_{34}\right)-\sin \left(2 \Delta_{35}\right)\right\},
\end{aligned}
$$




$$
P_{54}=\left(2 \epsilon-\epsilon^{2}\right)^{2} \sin ^{2}\left(2 \eta_{2}\right) \sin ^{2} \Delta_{45}
$$

Here $a=+1$ for neutrinos and $a=-1$ for antineutrinos.

1 S. M. Bilenky and B. M. Pontekorvo, Sov. Physics-Uspekhi 20, 776 (1977).

2 Particle Data Group (M. Tanabashi et al.), Phys. Rev. D 98, 030001 (2018).

3 I. Esteban, M. C. Gonzalez-Garcia, M. Maltoni, I. Martinez-Soler and T. Schwetz, J. High Energy Phys. 1701, 087 (2017).

4 V. V. Khruschov, S. V. Fomichev and O. A. Titov, Phys. Atom. Nucl. 79, 708 (2016) arXiv:1612.06544v1 [hep-ph]).

5 S. T. Petcov, I. Girardi and A. V. Titov, Int. J. Mod. Phys. A 30, 1530035 (2015).

6 The T2K Collaboration (K. Abe et al.), Phys. Rev. D 96, 092006 (2017) [Erratum: ibid. 98, 019902(E) (2018)].

7 S. Wang, Y.-F. Wang and D.-M. Xia, Chin. Phys. C 42, 065103 (2018).

8 LSND Collaboration (C. Athanassopoulos et al.), Phys. Rev. Lett. 77, 3082 (1996).

9 LSND Collaboration (A. Aguilar et al.), Phys. Rev. D 64, 112007 (2001).

10 MiniBooNE Collaboration (A. A. Aguilar-Arevalo et al.), Phys. Rev. Lett. 110, 161801 (2013).

11 MiniBooNE Collaboration (A. A. Aguilar-Arevalo et al.), Phys. Rev. Lett. 121, 221801 (2018).

12 Th. A. Mueller et al., Phys. Rev. C 83, 054615 (2011).

13 G. Mention et al., Phys. Rev. C 83, 073006 (2011).

14 P. Huber, Phys. Rev. C 84, 024617 (2011) [Erratum: ibid. 85, 029901(E) (2012)].

15 Y. J. Ko et al. (NEOS), Phys. Rev. Lett. 118, 121802 (2017).

16 I. Alekseev et al. (DANSS), Phys. Lett. B 787, 56 (2018).

17 A. P. Serebrov et al., arXiv:1809.10561v1 [hep-ex].

18 SAGE Collaboration (J. N. Abdurashitov et al.), Phys. Rev. C 80, 015807 (2009).

19 F. Kaether, W. Hampel, G. Heusser, J. Kiko and T. Kirsten, Phys. Lett. B 685, 47 (2010).

20 C. Giunti, M. Laveder, Y. F. Li and H. W. Long, Phys. Rev. D 88, 073008 (2013).

21 M. Vogelsberger et al., Nature 509, 177 (2014).

22 M. Demiański and A. G. Doroshkevich, arXiv:1511.07989v6 [astro-ph.CO].

${ }^{23}$ K. N. Abazajian et al., arXiv:1204.5379 [hep-ph].

24 T. Abe, R. Kitano and R. Sato, Phys. Rev. D 91, 095004 (2017).

25 A. Kusenko, Phys. Rep. 481, 1 (2009).

26 Y. Liao, Nucl. Phys. B 749, 153 (2006).

27 A. de Gouvêa, Phys. Rev. D 72, 033005 (2005).

28 D. S. Gorbunov, Physics-Uspekhi 57, 503 (2014).

29 M. Drewes and B. Garbrecht, Nucl. Phys. B 921, 250 (2017). 
30 Planck Collaboration (P. A. R. Ade et al.), Astronomy \& Astrophysics 571, A16 (2014).

31 K. N. Abazajian, Phys. Rep. 711712, 1 (2017).

32 M. Gerbino and M. Lattanzi, Frontiers in Phys. 5, 70 (2018) [doi:10.3389/fphy.2017.00070].

33 S. Hannestad, R. S. Hansen and T. Tram, Phys. Rev. Lett. 112, 031802 (2014).

34 X. Chu, B. Dasgupta and J. Kopp, J. Cosmol. Astropart. Phys. 1510, 011 (2015).

35 V. N. Gavrin, B. T. Cleveland, V. V. Gorbachev, T. V. Ibragimova, A. V. Kalikhov, Yu. P. Kozlova, I. N. Mirmov, A. A. Shikhin and E. P. Veretenkin, Phys. Part. Nucl. 48, 967 (2017).

36 V. Barinov, B. Cleveland, V. Gavrin, D. Gorbunov and T. Ibragimova, Phys. Rev. D 97, 073001 (2018).

37 G. Bellini et al., J. High Energy Phys. 1308, 038 (2013).

38 A. P. Serebrov et al., JETP Letters 109, 213 (2019).

39 T. Marrodán Undagoitia and L. Rauch, J. Phys. G: Nucl. Part. Phys. 43, 013001 (2016).

40 C. R. Argüelles, A. Krut, J. A. Rueda and R. Ruffini, Phys. Dark Universe 21, 82 (2018).

41 L. Canetti, M. Drewes and M. Shaposhnikov, Phys. Rev. Lett. 110, 061801 (2013).

42 J. M. Conrad, C. M. Ignarra, G. Karagiorgi, M. H. Shaevitz and J. Spitz, Adv. High Energy Phys. 2013, 163897 (2013).

43 N. Yu. Zysina, S. V. Fomichev and V. V. Khruschov, Phys. Atom. Nucl. 77, 890 (2014).

44 V. V. Khruschov and S. V. Fomichev, Phys. Part. Nucl. 48, 990 (2017).

45 V. V. Khruschov, A. V. Yudin, D. K. Nadyozhin and S. V. Fomichev, Astron. Lett. 41, 260 (2015).

46 M. L. Warren, G. J. Mathews, M. Meixner, J. Hidaka and T. Kajino, Int. J. Mod. Phys. A 31, 1650137 (2016).

47 A. V. Yudin, D. K. Nadyozhin, V. V. Khruschov and S. V. Fomichev, Astron. Lett. 42, 800 (2016).

48 T. Schwetz, M. Tórtola and J. W. F. Valle, New J. Phys. 13, 063004 (2011).

49 J. Kopp, P. A. N. Machado, M. Maltoni and T. Schwetz, J. High Energy Phys. 1305, 050 (2013).

50 S. Gariazzo, C. Giunti, M. Laveder and Y. F. Lie, J. High Energy Phys. 1706, 135 (2017).

51 S. M. Bilenky, Phys. Part. Nucl. Lett. 12, 453 (2015) (arXiv:1502.06158 [hep-ph]).

52 V. V. Khruschov and S. V. Fomichev, J. Phys.: Conf. Ser. 934, 012007 (2017).

53 E. Bulbul, M. Markevitch, A. Foster, R. K. Smith, M. Loewenstein and S. W. Randall, Astrophys. J. 789, 13 (2014).

54 A. Boyarsky, O. Ruchayskiy, D. Iakubovskyi and J. Franse, Phys. Rev. Lett. 113, 251301 (2014).

55 S. Horiuchi, B. Bozek, K. N. Abazajian, M. Boylan-Kolchin, J. S. Bullock, S. Garrison-Kimmel and J. Onorbe, Monthly Notices of the Royal Astro. Soc. 456, 4346 (2016).

56 M. Dutra, M. Lindner, S. Profumo, F. S. Queiroz, W. Rodejohann and C. Siqueira, J. Cosmol. Astropart. Phys. 1803, 037 (2018).

57 M. Blennow and A. Yu. Smirnov, Adv. High Energy Phys. 2013, 972485 (2013).

58 E. Akhmedov, J. Kopp and M. Lindner, J. Cosmol. Astropart. Phys. 1709, 017 (2017).

59 S. Gariazzo, C. Giunti, M. Laveder, Y. F. Li and E. M. Zavanin, J. Phys. G: Nucl. Part. Phys. 43, $033001(2015)$. 
60 Y. Kudenko and D. Wark, J. Phys.: Conf. Ser. 934, 012001 (2017).

61 M. Maltoni and T. Schwetz, Phys. Rev. D 76, 093005 (2007).

62 S. Palomares-Ruiz, S. Pascoli and T. Schwetz, J. High Energy Phys. 0509, 048 (2005).

63 G. Karagiorgi, A. Aguilar-Arevalo, J. M. Conrad, M. H. Shaevitz, K. Whisnant, M. Sorel and V. Barger, Phys. Rev. D 75, 013011 (2007) [Erratum: ibid. 80, 099902(E) (2009)].

64 M. Dentler, Á. Hernández-Cabezudo, J. Kopp, P. Machado, M. Maltoni, I. Martinez-Soler and T. Schwetz, J. High Energy Phys. 1808, 010 (2018).

65 M. Danilov, arXiv:1812.04085 [hep-ex].

66 S. Gariazzo, C. Giunti, M. Laveder and Y. F. Li, Phys. Lett. B 782, 13 (2018).

67 C. Giunti and T. Lasserre, arXiv:1901.08330 [hep-ph].

68 S. K. Kang, arXiv:1904.07108 [hep-ph]. 